\title{
Integrated mRNA and microRNA transcriptomic analysis reveals the common and individual responses of zebrafish embryos exposed to four types of graphene quantum dots (GQDs)
}

Shun Deng ( $\nabla$ dshun1979@swu.edu.cn )

Sichuan Provincial Orthopedic Hospital https://orcid.org/0000-0002-2565-6538

Enming Zhang

Beijing Sport University

Yunyang Zhao

University of Macau

\section{Yan Wang}

Chinese Academy of Sciences

\section{Zezhong Yang}

Tianjin Academy of Agricultural Sciences

Baiming Liu

Tianjin Academy of Agricultural Sciences

Li Liu

Chinese Academy of Sciences

Bingxin Zheng

Sichuan Provincial Orthopedic Hospital

Xiaoyuan Mu

Sichuan Provincial Orthopedic Hospital

Kezhu Yuan

Sichuan Provincial Orthopedic Hospital

\section{Xuangen Deng}

Sichuan Provincial Orthopedic Hospital

\section{Hai Shen}

Sichuan Provincial Orthopedic Hospital

Haibo Rong

Hospital Provincial

Desheng Pei

Chinese Academy of Sciences

Ailing Fu

Southwest University 


\section{Xia Li}

Hospital Provincial

\section{Research Article}

Keywords: Graphene quantum dots, Toxicity, Zebrafish, Transcriptomic response

Posted Date: June 9th, 2021

DOl: https://doi.org/10.21203/rs.3.rs-604090/v1

License: (c) (1) This work is licensed under a Creative Commons Attribution 4.0 International License. Read Full License 


\section{Abstract}

Background: Graphene quantum dots (GQDs) have great potential for bioimaging, biosensor, drug carrier, theranostics, and are recently reported as therapeutic agents to treat amylosis and inflammation. Most types of GQDs have proven low toxicity in previous studies, but data about the transcriptomic responses of in vivo systems exposed to various GQDs remains largely unknown.

Results: We examined the mRNA and microRNA (miRNA) expression changes of zebrafish embryos exposed to four types of GQDs at a safe concentration $(100 \mu \mathrm{g} / \mathrm{mL})$, respectively as raw graphene quantum dots (R-GQDs), graphene oxide quantum dots (GOQDs), carboxyl GQDs (C-GQDs), and aminated GQDs (A-GQDs). The four GQDs elicited the number of differentially expressed genes (DEGs) in a decreasing order of A-GQDs, GOQDs, C-GQDs and R-GQDs to act on protein folding, potassium $\left(\mathrm{K}^{+}\right)$and calcium $\left(\mathrm{Ca}^{2+}\right)$ channels, and spliceosome to varying degrees. Among the four GQDs, A-GQDs caused more genotoxic effects associated with lipid and hormone metabolism, MAPK signaling pathway, complement system, and ferropotosis. miRNA-seq data revealed that GOQDs aroused more differentially expressed miRNAs (DEMs), far exceed the total number of DEMs induced by the other three GQDs. dremiR-735-5p and its potentially interactive gene-myogenin (myog) were identified as the only negativelycorrelated miRNA-target gene pair shared by the four GQDs treatments.

Conclusion: Taken together, this study provided substantial data underlying the common and specific transcriptomic responses of in vivo systems exposed to various types of GQDs, and also indicated the potential medicinal values of GQDs for ion antagonists and spliceosome-targeted therapies.

\section{Background}

Graphene quantum dots (GQDs) are newly-emerged graphene-derived nanoparticles (NPs) with lateral dimensions typically below $10 \mathrm{~nm}$ in sizes, and show better performance in electronic, optical, spin, and photoelectric properties induced by the quantum confinement effect and edge effect [1]. GQDs hold promise in biomedical applications for their high biocompatibilities [2], tunable photoluminescence (PL) [3], light-dependent anti- (pro-) oxidant activities [4], and intrinsic peroxidase-like catalytic activities [5, 6], which have been exploited as sensitive fluorophores for real-time bioimaging and anticancer photodynamic therapy in in vivo systems [7-9], and also independently used for anti-inflammation via their strong free radical scavenging activities [10-12]. Interestingly, two recent studies found that GQDs could specifically disaggregate amyloid proteins (i.e. a-synuclein fibrils, islet amyloid polypeptide (IAPP)) and reduce the neurological toxicity in Parkinson's disease as well as improve IAAP-related symptoms [13, 14]. Although most studies claimed that GQDs are well biocompatible and induce low toxicity in organisms, there remain a few inconsistent findings regarding the potential toxicity of GQDs that may result from nanofabrication methods and the varying surface characters, including nanosizes, surface charges, functional groups, element doping, and impurities $[15,16]$. It is now generally believed that hydroxylated GQDs (OH-GQDs) are hazardous for its inducibility of oxidative stress even at relatively low exposure concentrations, leading DNA damage, $G_{0} / G_{1}$ cell cycle arrest, the disruption of microtubule 
structure, enhanced intestinal penetrability, and activation of multiple signaling pathways [17-19]. At present, nitrogen-doped GQDs (N-GQDs) and aminated GQDs (A-GQDs) have been investigated much for their toxicity, although some previous studies asserted that they were low toxic to cell survival and animal development [18, 20-22]. Most toxic data about the two GQDs were evidenced from their effects on cellular micromorphology, nucleotide structures, and gene expression profiling. Both were found to induce DNA methylation and transcriptomic responses in relation to the canonical MAPK signaling pathway [23-26]. In addition, it was reported that N-GQDs could deform the structure of lipid droplets, disrupt the redox-sensitive system, and cause ferroptosis [27-29], while A-GQDs could induce DNA cleavage via $\mathrm{H}$ bonding and $\pi-\pi$ stacking [30]. In general, the low toxicity of most types of GQDs was concluded from the conventional toxicity testing, involving the assessment of cell growth and survival, the developmental indices in animal models, and the canonical blood/molecular markers [22, 31-33], and there remains incomplete whether all GQDs share the common toxic pathways and to what extent various types of GQDs perturb specific responses of genes, proteins, metabolites, as well as the behaviors. Now there have been reported several studies as mentioned above that employed toxicomics approaches to unveil the potential genotoxicity caused by GQDs [24-26], but the relevant toxicity assessment of GQDs using highthroughput screening methods based on in vivo systems are seldom [24].

Zebrafish as an ideal animal model has been extensively used in the biosafety evaluation of a wide range of engineered nanomaterials (ENMs) [34]. This animal possesses a suit of conserved evolutionary system that is comparable to that in vertebrates and has developed a typical optical-transparency body at early stage that is easily observed when undergoing phenotypic variations caused by genetic changes, environmental harmful factors, and among others [35]. Compared to the mouse model, zebrafish exhibits unique characteristic in its high-fecundity that provides considerable number of embryos for highthroughput drug screening [36]. In addition, the fish is low-cost reared and easily genetically manipulated in sizes. Zebrafish has been widely applied for the toxicity assessment of a series of carbon-based nanomaterials, including carbon nanotubes (CNTs) [37], carbon fullerene (mainly C60) [38], graphene (G) [39], graphene oxide (GO) [40], carbon quantum dots (CQDs) [41], and N-GQDs [28, 42]. In the study, we employed high-throughput sequencing technology to explore the potential side effects of four types of GQDs on in vivo transcriptional profiling as opposed to gene selection based on known markers in canonical toxicity assays. The study aimed to reveal the common gene clusters and associated signaling pathways induced by the four GQDs in in vivo systems, and also pointed out the specific pathway and the miRNA-mRNA interaction triggered by different GQDs. This study would provide valuable insights about the potential genotoxicity of various GQDs on in vivo systems, and also an alert for the selection of GQDs especially for pharmacotherapies.

\section{Methods}

\section{Nanoparticles preparation and surface characterization}

Four commercially GQDs were purchased from Nanjing XFNANO Materials Tech Co., Ltd (XFNANO), respectively as graphene quantum dots (R-GQDs, XF215), graphene oxide quantum dots (GOQDs, XF074), 
carboxylated GQDs (C-GQDs, XF090), and aminated GQDs (A-GQDs, XF092), all of which have been reported for biosafety assessment and drug therapy in previous studies $[10,18,22,23,25,26,29,30]$. Except GOQDs made from top-down method, the other three GQDs were all fabricated using bottom-up method.TEM images of the four GQDs were obtained by a Transmission Electron Microscope (Talos F200X, FEI, USA) under the operating voltage of $200 \mathrm{kV}$. XPS data of four GQDs were acquired from Escalab 250Xi (Thermo Fisher Scientific, USA). The surface charges of four GQDs in three mediums (ultrapure water $\left(\mathrm{ddH}_{2} \mathrm{O}\right), 1 \times \mathrm{PBS}(\mathrm{pH} 7.4, \mathrm{Gibco})$, and $1 \times \mathrm{DMEM}$ (Gibco)) were measured using the Zetasizer Nano ZS apparatus (Malvern Instruments Ltd, Malvern, U.K.)

\section{Zebrafish Husbandry And Exposure Assays}

From the larval stage, a batch of wild-type zebrafish $(A B)$ were raised according to the standard breeding protocol [43]. Zebrafish at about 4 months old were coupled for spawning and the newly-laid eggs were collected at four post hour fertilization. Fifty embryos were slightly pipetted into every well of 6-well cell plates filled with $3 \mathrm{~mL}$ various GQDs solutions $(100 \mu \mathrm{g} / \mathrm{mL})$, which was newly prepared every day. Each treatment was performed for at least four biological replicates, and control group was only treated with water. The survival rates of zebrafish embryos exposed to different GQDs were recorded at 2 day post fertilization (dpf) until to the end of the assay. Hatching rate was estimated at $2 \mathrm{dpf}$ and $3 \mathrm{dpf}$, and heart rate (beats/min) was observed at $3 \mathrm{dpf}$, 4dpf, and 5dpf using an inverted microscope (Nikon SMZ18, Japan), each treatment was carried out with at least three independent biological replicates. After exposure for $7 d$, zebrafish embryos were collected from each well and kept at $-80^{\circ} \mathrm{C}$ refrigerator.

\section{MRNA And MIRNA Extraction And Sequencing}

Total RNAs were extracted from zebrafish embryos using TRIzol® Reagent according to the manufacturer's instruction (Invitrogen). Genomic DNA debris was degraded using DNase I RNase-free (Takara, Japan). RNA quality was evaluated on $1 \%$ agarose gels and its concentration was measured by means of Nanodrop-2000 (Thermo Fisher Scientific, USA). Finally, RNA integrity was monitored using a 2100 Bioanalyzer (Agilent Technologies, Santa Clara, CA, USA), and only high-quality RNA samples (OD260/280 = $1.8 \sim 2.2$, OD260/230 $\geq 2.0$, RIN $\geq 7,28 S: 18 S \geq 1.0$, quantity $>5 \mu \mathrm{g}$ ) were applied for constructing sequencing libraries. (1) mRNA sequencing: RNA-seq transcriptome library was prepared following TruSeqTM RNA sample preparation Kit from Illumina (San Diego, CA) using $1 \mu \mathrm{g}$ of total RNA. Shortly, messenger RNA was isolated according to polyA selection method by oligo (dT) beads and then fragmented by fragmentation buffer firstly. Secondly, double-stranded cDNA was synthesized using a SuperScript double-stranded cDNA synthesis kit (Invitrogen, CA) with random hexamer primers (Illumina). Then the synthesized cDNA was subjected to end-repair, phosphorylation and ' $A$ ' base addition according to Illumina's library construction protocol. Libraries were size selected for CDNA target fragments of 300 bp on 2\% Low Range Ultra Agarose followed by PCR amplified using Phusion DNA polymerase (NEB) for 15 PCR cycles. After quantified by TBS380, paired-end RNA-seq sequencing library was sequenced with the Illumina HiSeq xten/NovaSeq 6000 sequencer ( $2 \times 150 \mathrm{bp}$ read length). (2) miRNA sequencing: RNAseq transcriptome library was prepared following TruSeq ${ }^{\text {TM }}$ RNA sample preparation Kit from Illumina (San Diego, CA) using $1 \mu \mathrm{g}$ of total RNA. Shortly, messenger RNA was isolated according to polyA 
selection method by oligo(dT) beads and then fragmented by fragmentation buffer firstly. Secondly double-stranded cDNA was synthesized using a SuperScript double-stranded cDNA synthesis kit (Invitrogen, CA) with random hexamer primers (Illumina). Then the synthesized cDNA was subjected to end-repair, phosphorylation and ' $A$ ' base addition according to Illumina's library construction protocol. Libraries were size selected for CDNA target fragments of 300 bp on 2\% Low Range Ultra Agarose followed by PCR amplified using Phusion DNA polymerase (NEB) for 15 PCR cycles. After quantified by TBS380, paired-end RNA-seq sequencing library was sequenced with the Illumina HiSeq xten/NovaSeq 6000 sequencer $(2 \times 150 \mathrm{bp}$ read length).

\section{Bioinformatics Analysis}

Principal component analysis (PCA) was performed for mRNAs and miRNAs data with OmicShare tools, a free online platform for data analysis (http://www.omicshare.com/tools). The fragments per kilobase million (FPKM) and transcripts per million (TPM) values were employed to quantify mRNA and miRNA expression levels, respectively. The differentially expressed genes (DEGs) and differentially expressed miRNAs (DEMs) were screened out using DESeq2. Gene ontology (GO) and KEGG pathways of DEGs were analyzed with the online DAVID Bioinformatics Resources 6.8 and KOBAS 3.0 software, with a Bonferroni-corrected $P$-value $\leq 0.05$ compared with the whole-transcriptome background. Heatmap of DEGs and DEMs among different treatment was drawn using ggplot2. Protein-protein interaction (PPI) network was deduced by means of online String tool and Cytoscape (Version 3.8.0). Through a BLAST search of the miRbase (version 21.0), the perfectly matched sequences were used to count and analyze the known miRNA expression profile. In-house scripts were used to obtain the identified miRNA base bias on the first position with certain length and on each position of all identified miRNA. The miRNA-target interactions were predicted by OmniSearch analysis that provides access to multiple target prediction dataset, including TargetSCan, miRanda, miRDB, and RNAhybrid. An index of corrected correlation $<-0.5$ (Spearman's correlation coefficients) was used to evaluate the negative correlations between known miRNA and target genes.

\section{Quantitative Realtime-PCR (QPCR) Verification}

The first-strand cDNA was transcribed with Fastking RT Kit with gDNase (KR116, TIANGEN, Beijing) and miRcute Plus miRNA First-Strand cDNA Kit (KR211, TIANGEN, Beijing) respectively for mRNAs and miRNAs according to the manufacturer's instructions. qPCR of mRNA and miRNA was performed on a Roche LightCycler 480 II (LC480, Roche Diagnostics) in a $20 \mu \mathrm{L}$ reaction. Primer pairs used for mRNA and miRNA were shown in Table S1. The relative mRNA and miRNA levels were respectively normalized to the internal control egfa and U6 snRNA and calculated using the $2^{-\Delta \Delta C t}$ method. Data were shown as Mean \pm SD from four independent biological replicates.

\section{Results}

Surface characters and developmental toxicity assessment 
Transmission Electron Microscopy (TEM) was used to characterize the morphology and size distribution of four kinds of GQDs. As shown in Fig. 1a, the TEM images showed that the four kinds of GQDs gave a narrow size distribution with well monodisperse, which indicated their high quality. Clear lattice fringes exhibited their graphitic structure with lattice spacing $(d=0.21 \mathrm{~nm}, 0.24 \mathrm{~nm})$ corresponding to $(100)$ diffraction facets of graphite [44-46]. Data of XPS full spectrum scan showed that GOQDs were among the most complex in the atomic ratio, including $\mathrm{C} 1 \mathrm{~s}(44.21 \%), 01 \mathrm{~s}(34.59 \%)$, Na1s (6.79\%), Si2p (8\%), $\mathrm{Cl} 2 \mathrm{p}(2.44 \%), \mathrm{S} 2 \mathrm{p}(2.28 \%)$, and Sc2p (6.11\%), whilst C-GQDs contained only C1s (58.13\%) and $01 \mathrm{~s}$ (41.87\%) (Fig. 1b and Table S2). We performed the curve fitting analysis based on the individual elemental scanning, mainly C1s and 01s (Fig. S1a). C1s spectra of R-GQDs and A-GQDs were deconvoluted into five peaks of C-C/C $=\mathrm{C}(\sim 284.6 \mathrm{eV}), \mathrm{C}-\mathrm{N}(\sim 285.7 \mathrm{eV}), \mathrm{C}-\mathrm{O}(\sim 286.6 \mathrm{eV}$, from C-O or C-OC), $\mathrm{C}=\mathrm{O}(\sim 288.0 \mathrm{eV})$, and $\mathrm{O}=\mathrm{C}-\mathrm{O}(\sim 288 \mathrm{eV})$. The percentage composition of $\mathrm{C}=\mathrm{O}$ was higher than that of $\mathrm{C}-\mathrm{O}$ in $\mathrm{A}-\mathrm{GQD}$ s, while R-GQDs showed the opposite compositional change of $\mathrm{C}=\mathrm{O}$ and $\mathrm{C}-\mathrm{O}$. Both GOQDs and C-GQDs were characteristic of four components in their $\mathrm{C} 1 \mathrm{~s}$ spectra, including $\mathrm{C}-\mathrm{C} / \mathrm{C}=\mathrm{C}, \mathrm{C}-\mathrm{O}$, $\mathrm{C}=\mathrm{O}$, and $\mathrm{O}=\mathrm{C}-\mathrm{O}$. The carboxyl $\mathrm{O}=\mathrm{C}-\mathrm{O}(\sim 533.6 \mathrm{eV})$ was also observed in the 01s profile of GOQDs and C-GQDs, along with $\mathrm{C}=0(\sim 531.5 \mathrm{eV})$ and $\mathrm{C}-\mathrm{O}(\sim 532.7 \mathrm{eV}$, from C-O or C-O-C), whilst the 01 s spectra of RGQDs and A-GQDs were only composed of $\mathrm{C}=\mathrm{O}$ and $\mathrm{C}-\mathrm{O}$. In addition, we made a XPS spectrum scan for the $\mathrm{N} 1 \mathrm{~s}$ profile of A-GQDs and it was divided into three strong peaks of $\mathrm{N}-\mathrm{H}(\sim 399.2 \mathrm{eV}), \mathrm{N}-\mathrm{C}=\mathrm{O}(\sim 399.7$ $\mathrm{eV})$, and $\mathrm{NH}_{\mathrm{n}}{ }^{+}(400.8 \mathrm{eV})$ (Fig.S1b), which were consistent with the previous report [30]. Next, it was found from the zeta potential analysis that the four GQDs were all negatively charged in three mediums (Fig.S1c), including ddH $\mathrm{H}_{2} \mathrm{O}, 1 \times$ PBS, and $1 \times$ DMEM. Among the four NPs, C-GQDs induced the weakest zeta potential changes in the three mediums, ranging from $-1 \sim-5 \mathrm{eV}$. The developmental characters of zebrafish embryos including survival rate, hatching rate, and hearbeat rate were not significantly changed by different GQDs treatment ( $100 \mu \mathrm{g} / \mathrm{mL}$ ) when compared to control group (Fig. S2: a-c). Our exposure assay manifested the low toxicity of the four GQDs to zebrafish embryonic development.

\section{Whole Transcriptomic Profiling}

Principle component analysis (PCA) revealed that the mRNA expression data extracted from GOQDs and C-GQDs treatment clustered in close distances, as well as the known miRNA sequencing data among the clusters of control, R-GQDs, and C-GQDs treated groups. A-GQDs treatment was clearly separated from the others regardless of mRNA or miRNA data (Fig. $2 a$ and $2 b$ ), and the miRNA data from GOQDs treatment also clustered independently. With the same corrected $p$ value $(F D R)<0.05$ and two cutoff settings ( $F C>2$ or 1.5), two ensembles of DEGs were screened out between control and NPs treated groups. A-GQDs elicited the largest number of DEGs (FC > 2, 1,878; FC > 1.5, 4,796), far more than the total number of DEGs induced in other three GQDs exposed groups (Fig. 2c, Table S3). However, from the miRNA expression levels, GOQDs were found to induce more DEMs (FC > 2, 32; FC > 1.5, 65) than other GQDs, and R-GQDs and C-GQDs exerted negligible effects on miRNAs expression levels (Fig. 2c, Table S4). To excavate the potential genes commonly induced by different GQDs, the Venn analysis based on the DEGs and DEMs data from respective GQDs treatment was performed with a FC cutoff $>1.5$ and a 
significant $F D R$ value of $<0.05$, and 75 DEGs were identified to be shared by the four GQDs treatments (Fig. 2d, Table S5), and no overlapping miRNAs are found among various GQDs treatments.

\section{Data Profiling Of DEGs}

Conventionally, we performed the functional enrichment analysis of Gene Ontology (GO) and Kyoto Encyclopedia of Genes and Genomes (KEGG) pathways for DEGs identified from different GQDs treatment. With the setting of the threshold as $F D R<0.05$, more than 500 subcategories were significantly enriched into three divisions in A-GQDs treated group, encompassing biological process (BP, 329 terms), cellular component (CC, 86 terms), and molecular function (MF, 91 terms). Comparatively, the significantly-enriched GO items were less found in C-GQDs (66), GOQDs (48), and R-GQDs treated group (48) (Table S6). In the top ten GO terms from A-GQDs and GOQDs groups, both share two identical GO terms - 'cellular response to unfolded protein and response to unfolded protein' (Fig. 3a), from which DEGs related to hsp genes are highly upregulated. Compared to other GQDs treatments (Fig. 3b), A-GQDs treatment induced more KEGG pathways in relation to lipid and hormone metabolism (Fig. S3a), MAPK signaling pathway (Fig. S3b), complement system (Fig. S3c), ferropotosis (Fig. S3d), cytochrome P450 system (Fig. S3e), all of which directly or indirectly interacted with each other and finally triggered the (pro-) apoptotic signals (Fig. S4).

The up-regulation of $h s p$ genes usually signals the organisms suffering stressful environmental conditions and their translated proteins (heat shock proteins, HSPs) act as intra-cellular chaperones to stabilize novel proteins by guiding their proper folding and conformation [47], we herein made a generalization for all $h s p$ genes and relevant genes across the mRNA expression profiling in all treatments. Heatmap displayed that A-GQDs induce the most number of $h s p$ and associated genes, and R-GQDs elicited the minimal transcriptomic responses of HSP family members (Fig. 4a). To validate the RNA-seq data, ten $h s p$ genes were selected from the Heatmap for qPCR amplification. Results confirmed that most of the qPCR data were in accordance with the mRNA-seq results, and hspa4a, hsp70.2, $h s p 70.3$, and $h s p 90 a a 1.2$ were significantly upregulated in the four GQDs treatments when compared to controls (Fig. 4b).

To better explore DEGs and associated signaling pathways commonly aroused by the four GQDs, we firstly performed a Venn analysis based on the significantly enriched GO terms from different GQDs treatment, and two GO terms - 'ion membrane transport and ion transport' are found to be shared by the four GQDs treatments (Fig. 5a). The further Venn analysis revealed that 6 and 10 DEGs were shared by the four GQDs in each GO term (Fig. 5b and Fig. 5c), and finally 8 genes were determined from these DEGs (Fig. 5d). Except the upregulated expression of apoa4b. 2 in the four GQDs treatments, other genes related to potassium $\left(\mathrm{K}^{+}\right)$and calcium $\left(\mathrm{Ca}^{2+}\right)$ channels were significantly suppressed by the four GQDs. We further made a generalization about the overlapping 75 DEGs among the four GQDs treatments, which exhibited a congruous expression profiling within three repeating samples between control and treatment groups (Fig. 5e). GO enrichment analysis showed that the overlapping genes were significantly enriched into protein $\mathrm{N}$-acetylglucosaminyltransferase and RNA processing, and splicesome was 
identified as the only significantly enriched KEGG pathway (Table S7). Next, we performed the proteinprotein interactions (PPI) analysis based on these overlapping genes and showed that serine/argininerich splicing factor 11 (srfs11) as the center mediator coordinating with other splicing factor to regulate downstream genes, including $n k t r$, hspbp1, rbm5, gpatch8, and arglu1a (Fig. 5f).

\section{Data Profiling Of MIRNAs}

In the study, the target genes of DEMs in different GQDs treatment were screened out using multiple prediction databases with reference to respective DEGs database, from which a total of 148 and 153 coexpressed genes for 52 and 13 miRNAs were respectively identified from GOQDs and A-GQDs treatments, and was few miRNA-mRNA pairs found in the other two treatments. Owing to very few GO and KEGG pathways significantly enriched from the target DEGs $(F D R<0.05)$, we focused on the data mining of the miRNA-mRNA pairs with negative correlations from the four GQDs treatments. 86 and 42 anti-correlated miRNA-mRNA pairs were identified from GOQDs and A-GQDs treated groups, respectively extracted from the combination of 43 miRNAs: 65 genes in GOQDs and 11 miRNAs: 36 genes in A-GQDs treatment, while only one negative miRNA-mRNA pair was identified from the other two treatments (Table S8). Together with the above generalization of the common signaling pathways induced by the four GQDs, several pairs from GOQDs likely involved in the ion membrane transport, ion transport, and the spliceosome pathway. From the GOQDs treatment, three genes (s/c25a23a, kcnn1a, trpm1b) implicating in the ion membrane transport and ion transport' were predicted to be repressed by four miRNAs (dre-miR-455-2-5p, dre-miR140-5p, 10_1985, 24_18026), while three genes (srsf2a, srsf5a, srsf5a) involving in the spliceosome pathway were probably negatively regulated by dre-miR-15a-5p and dre-miR-16a. In addition, three genes (socs1a, il6st, map2k6) involving in the MAPK signaling pathways were identified to be repressed by dremiR-7132-3p, dre-miR-19a-5p, and dre-miR-216a in GOQDs treatment. dre-miR-29b3-3p from A-GQDs treatment showed the strongest multitargeting activities and likely involved in the downregulated the mRNA levels of nine genes, in which two pairs (dre-miR-29b3-3p: s/c38a8b and dre-miR-29b3-3p: slc44a2) may collaboratively act on the splicesome pathway.

From all negatively correlated miRNA-mRNA pairs, we found that the pair of dre-miR-735-5p and its negatively regulated gene - myogenin (myog) was shared by A-GQDs, GOQDs, and C-GQDs treated groups, and dre-miR-735-5p was not significantly up-regulated by R-GQDs according to the originally-used screening threshold $(F D R<0.5, \mathrm{FC}>1.5)$. However, it was found that dre-miR-735-5p was induced more than three-fold change (3.165) in R-GQDs treated group when the cutoff as set as $p$ value $<0.01$ and FC > 1.5. Then, we employed qPCR to investigate the expression changes of dre-miR-735-5p and its target gene-myog among the four GQDs treatments, and results showed that the expression levels of dre-miR735-5p in all GQDs treatments were significantly activated when compared to control group, and myog exhibited the similar expression profiling to that in RNA-seq data (Fig. 6), and then suggesting that the dre-miR-735-5p-myog pair could be commonly aroused by the four GQDs.

\section{Discussion}


GQDs are typical of graphene structure with honeycomb lattices and inherit many physicochemical properties from graphene. GQDs develop strong photoluminescence $(\mathrm{PL})$ and photostability with highly tunable band gaps, which have been exploited for sensitive fluorophores to monitor living cells and drug carriers to assist target therapy in in vivo systems. More strikingly, several recent studies showed that GQDs can be independently used as pharmaceutical agents to treat inflammations and amylosis [13, 14], thereby being paid much attention for its potential medicinal value. However, GQDs are diversely characterized by the fabrication method and raw material, thus leading to the potential nano-bio effects that may be unsafe [48]. Therefore, the systemic biosafety assessment of GQDs would be demand before its approval for clinical trials. In the previous study, we had examined the transcriptomic responses (mRNAs) of zebrafish embryos exposed to N-GQDs, which were found to trigger pathways mainly associated with acute inflammation and detoxifying process [24]. In the study, we extended the number of GQDs for toxicity evaluation, and the four types of GQDs including R-GQDs, GOQDs, C-GQDs, and AGQDs were the first time to be investigated for their potential genotoxic effects based on in vivo systems. Our exposure assay exhibited the low toxicity of the four GQDs on the developmental characters of zebrafish embryos, some of which was also observed in mice treated with the same four GQDs [22], and then suggesting that the adverse effects induced by GQDs would not be easily discriminated upon the conventional toxicity assays such as the assessment of cell growth and survival, the developmental indices in animal models, and the canonical blood/molecular markers. Thus, the employment of highthroughput transcriptomics would be an optimal choice to unravel the potential genotoxic effects induced by various types of GQDs. Our study showed that the four GQDs can act on unfolded protein responses and endoplasmic reticulum via activating the HSPs family members and associated pathways to varying degrees, then suggesting that GQDs may cause dysfunction of certain proteins, but the process of which would be counteracted by a group of HSP family members mobilized by GQDs. Previous studies showed that GQDs can break down amyloid oligomerization and are promised as potential small molecules for treating amylosis $[13,14]$, and then whether GQDs specifically target amyloid proteins or not remains to be explored in the future.

Human body is driven by a complex electric circuit comprising finely tuned and intricately synchronized charge transfer systems, which are the principal drive for the flows of energy to sustain the vital activities, such as the Krebs cycle (citric acid or tricarboxylic acid cycle) in aerobic organisms, being a singledirection cyclic pathway transferring electrons from citrate to NAD ${ }^{+}, F A D$ and GDP to produce NADH, $\mathrm{FADH}_{2}$, and GTP [49]. Importantly, the electron transferring serves as bidirectional messengers in the redox homeostasis, which maintains the normal physiological steady state in living organisms [50]. GQDs show metal-like attributes and have been reported to possess anti- and pro-oxidant activities [4], and our functional enrichment analysis revealed that the four GQDs can disturb $\mathrm{K}^{+}$and $\mathrm{Ca}^{2+}$ homeostasis by co-suppressing $\mathrm{K}^{+}$and $\mathrm{Ca}^{2+}$ channel associated gene clusters (Fig. $5 \mathrm{C}$ ), then suggesting that GQDs may involve in the metal homeostasis via energy and charge transfer. The inhibitory effects of GQDs on $\mathrm{K}^{+}$and $\mathrm{Ca}^{2+}$ channel were also observed in the mouse lung and the primary cortical neurons of rats treated with graphene oxide nanosheets $[51,52]$. Blocking $\mathrm{K}^{+}$and $\mathrm{Ca}^{2+}$ channel can jointly or independently delay the action potential repolarization and intensify the blood vessel dilatation, and 
sometimes trigger irregular physiological responses in muscle and cardiovascular system [53, 54]. However, this impeding effect has been often reported to play active roles in treating arrhythmia [55], hypoglycemia [56], and hyperglycemia [57], thus meaning GQDs could be potential ion channel antagonists to treat relevant diseases. Therefore, the inhibitory effects of GQDs on $\mathrm{K}^{+}$and $\mathrm{Ca}^{2+}$ channel should be adequately evaluated by combing with other physiological responses. Our finding about the transcriptional regulation of spliceosome triggered by GQDs was also reported in A549 cell line and zebrafish treated with $\mathrm{GO}[58,59]$, implying that the pathway could be commonly aroused by GQDs and other graphene derivatives. Splicing factors such as splicing factors $3 \mathrm{~b}$ (SF3B) have been reported as a core target for anticancer treatment [60], and in this study five splicing factors (srsf2a, sf3b1, sif4, srsf5a, and $z g c:$ 163098) from the spliceosome pathway were all significantly downregulated by the four GQDs, indicating the potential of GQDs as spliceosome inhibitors or adjuvants for targeted cancer therapy.

In this study, the number of miRNAs triggered by GOQDs was much more than that of other three GQDs. However, the miRNA clusters and associated miRNAs-mRNAs regulatory network induced by GOQDs were totally different from that aroused by $G O$ in in vivo system [61, 62], suggesting that the regulatory effects of GOQDs on miRNAs have been developed independently from that of $G$ and GO. Still, a few miRNAmRNA pairs identified from GOQDs treatment may involve in MAPK signaling pathway (Table S7). Although the miRNA responses were weak relative to the mRNA responses induced by GQDs, a negatively regulated miRNA-mRNA (dre-miR-735-5p: myog) was found to be aroused by the four GQDs. Myogenin (myog) is reported as the key regulator for the proper differentiation of most myogenic precursor cells during the process of myogenesis [63], indicating that GQDs may preclude the development of functional skeletal muscle via the commonly activation of dre-miR-735-5p and its target gene-myog.

Among the four GQDs, A-GQDs showed more genotoxic effects on in vivo system compared to other GQDs, and most of the toxic pathways aroused by A-GQDs were also identified in N-GQDs treated zebrafish embryos [24], including the complement and coagulation system, MAPK pathway, cytochrome P450 system, lipid and hormone metabolism, etc. Interestingly, we found that A-GQDs and N-GQDs shared the similar N1s profile in two peaks corresponding with amide bond and ammonium ions [42]. Together with the previous finding about the disruptive effects of A-GQDs on DNA chains [30], the high genotoxicity of A-GQDs may result from the enhancement of the polarization of A-GQDs caused by the lone pair electrons of the amino groups, which react with the phosphoric acid in nucleotides and disturb the structure of DNA bases. Therefore, A-GQDs should be functionally improved to lessen its potential genotoxic effects when used for the in vivo therapies.

\section{Conclusion}

In the present study, we confirmed that the four GQDs at the same exposure concentration $(100 \mu \mathrm{g} / \mathrm{mL})$ had no noticeably adverse effects on zebrafish embryonic development. Our microarray data was the first time to reveal that various types of GQDs act on at least three gene clusters in relation to protein folding, ion channels, and RNA splicing to varying degrees. A-GQDs were among the most genotoxic NPs for its perturbation of multiple signaling pathways, most of which were also identified from that in zebrafish 
exposed to N-GQDs. Although the miRNAs expression changes aroused by the four GQDs were much weaker than mRNAs responses, a negatively correlated miRNA-target gene pair (dre-miR-735-5p: myog) was identified to be commonly activated by the four GQDs, showing a common miRNA-mRNA regulatory network occurring among all GQDs. Our mRNA-seq data also provided invaluable information for the design of targeted drug delivery based on GQDs biocompatibility.

\section{Declarations}

\section{Ethics approval and consent to participate}

Animal experiments were carried out by stringently complying with the 'Guide for the Care and Use of Laboratory Animals' (Eighth Edition, 2011. ILARCLS, National Research Council, Washington, D.C.). The animal protocol was approved by the Animal Care and Use Committee of Chongqing in China and by the Institutional Animal Care and Use Committee of Chongqing Institute of Green and Intelligent Technology, Chinese Academy of Science (Approval ID: ZKCQY0510)

\section{Consent for publication}

The submission of this manuscript was approved by all authors.

\section{Competing interests}

All authors declare no competing interests.

\section{Funding}

This research was supported by Sichuan Medical Association (S20022) and Fundamental Research Funds for the Central Universities (2015CP001).

\section{Authors' contributions}

$\mathrm{SD}, \mathrm{HB}$, and HS designed the experiment. SD performed the transcriptomic data analysis and wrote the draft manuscript with EM. YY performed the surface characterization and data analysis. YW, LL, DS provided assistance in zebrafish husbandry, exposure assay, and sampling. ZZ and BM gave some suggestions in the transcriptomic data analysis. AL contributed some chemical reagents for this study. $B X, X Y, K Z, X G$, and $X L$ involved in the collection of some references and did the RT-PCR verification.

\section{Acknowledgement}

Not applicable.

\section{References}


1. Yan Y, Gong J, Chen J, Zeng Z, Huang W, Pu K, Liu J, Chen P. Recent Advances on Graphene Quantum Dots: From Chemistry and Physics to Applications. Adv Mater. 2019;31:e1808283.

2. Zhao X, Gao W, Zhang H, Qiu X, Luo Y. 20 - Graphene quantum dots in biomedical applications: recent advances and future challenges. In: Handbook of Nanomaterials in Analytical Chemistry. Edited by Mustansar Hussain C: Elsevier; 2020. pp. 493-505.

3. Lu Y, Hao H, Liu P, Feng Y, Wang J: Controllable synthesis of Graphene Quantum Dots with TunablePhotoluminescence. IOP Conference Series: Materials Science and Engineering 2020, 768:022073.

4. Chong Y, Ge C, Fang G, Tian X, Ma X, Wen T, Wamer WG, Chen C, Chai Z, Yin JJ. Crossover between Anti- and Pro-oxidant Activities of Graphene Quantum Dots in the Absence or Presence of Light. ACS Nano. 2016;10:8690-9.

5. Lin L, Song $X$, Chen Y, Rong M, Zhao T, Wang Y, Jiang Y, Chen X. Intrinsic peroxidase-like catalytic activity of nitrogen-doped graphene quantum dots and their application in the colorimetric detection of $\mathrm{H}_{2} \mathrm{O}_{2}$ and glucose. Anal Chim Acta. 2015;869:89-95.

6. Sun H, Zhao A, Gao N, Li K, Ren J, Qu X. Deciphering a nanocarbon-based artificial peroxidase: chemical identification of the catalytically active and substrate-binding sites on graphene quantum dots. Angew Chem Int Ed Engl. 2015;54:7176-80.

7. Zhang R, Ding Z. Recent Advances in Graphene Quantum Dots as Bioimaging Probes. Journal of Analysis Testing. 2018;2:45-60.

8. Lu H, Li W, Dong H, Wei M. Graphene Quantum Dots for Optical Bioimaging. Small. 2019;15:e1902136.

9. Ge J, Lan M, Zhou B, Liu W, Guo L, Wang H, Jia Q, Niu G, Huang X, Zhou H, et al. A graphene quantum dot photodynamic therapy agent with high singlet oxygen generation. Nat Commun. 2014;5:4596.

10. Ren C, Hu X, Zhou Q. Graphene Oxide Quantum Dots Reduce Oxidative Stress and Inhibit Neurotoxicity In Vitro and In Vivo through Catalase-Like Activity and Metabolic Regulation. Adv Sci (Weinh). 2018;5:1700595.

11. Tosic J, Stanojevic Z, Vidicevic S, Isakovic A, Ciric D, Martinovic T, Kravic-Stevovic T, Bumbasirevic V, Paunovic V, Jovanovic S, et al. Graphene quantum dots inhibit T cell-mediated neuroinflammation in rats. Neuropharmacology. 2019;146:95-108.

12. Lee BC, Lee JY, Kim J, Yoo JM, Kang I, Kim JJ, Shin N, Kim DJ, Choi SW, Kim D, et al. Graphene quantum dots as anti-inflammatory therapy for colitis. Sci Adv. 2020;6:eaaz2630.

13. Wang M, Sun Y, Cao X, Peng G, Javed I, Kakinen A, Davis TP, Lin S, Liu J, Ding F, Ke PC. Graphene quantum dots against human IAPP aggregation and toxicity in vivo. Nanoscale. 2018;10:1999520006.

14. Kim D, Yoo JM, Hwang H, Lee J, Lee SH, Yun SP, Park MJ, Lee M, Choi S, Kwon SH, et al. Graphene quantum dots prevent alpha-synucleinopathy in Parkinson's disease. Nat Nanotechnol. 2018;13:812-8.

15. Wang S, Cole IS, Li Q. The toxicity of graphene quantum dots. RSC Advances. 2016;6:89867-78. 
16. Ou L, Song B, Liang H, Liu J, Feng X, Deng B, Sun T, Shao L. Toxicity of graphene-family nanoparticles: a general review of the origins and mechanisms. Part Fibre Toxicol. 2016;13:57.

17. Li M, Gu MM, Tian X, Xiao BB, Lu S, Zhu W, Yu L, Shang ZF. Hydroxylated-Graphene Quantum Dots Induce DNA Damage and Disrupt Microtubule Structure in Human Esophageal Epithelial Cells. Toxicol Sci. 2018;164:339-52.

18. Xie Y, Wan B, Yang Y, Cui X, Xin Y, Guo LH. Cytotoxicity and autophagy induction by graphene quantum dots with different functional groups. J Environ Sci (China). 2019;77:198-209.

19. Yu L, Tian X, Gao D, Lang Y, Zhang XX, Yang C, Gu MM, Shi J, Zhou PK, Shang ZF. Oral administration of hydroxylated-graphene quantum dots induces intestinal injury accompanying the loss of intestinal stem cells and proliferative progenitor cells. Nanotoxicology. 2019;13:1409-21.

20. Liu Q, Guo B, Rao Z, Zhang B, Gong JR. Strong two-photon-induced fluorescence from photostable, biocompatible nitrogen-doped graphene quantum dots for cellular and deep-tissue imaging. Nano Lett. 2013;13:2436-41.

21. Zhao Y, Liu Q, Shakoor S, Gong JR, Wang D. Transgenerational safety of nitrogen-doped graphene quantum dots and the underlying cellular mechanism in Caenorhabditis elegans. Toxicology Research. 2015;4:270-80.

22. Li J, Zhang X, Jiang J, Wang Y, Jiang H, Zhang J, Nie X, Liu B. Systematic Assessment of the Toxicity and Potential Mechanism of Graphene Derivatives In Vitro and In Vivo. Toxicol Sci. 2019;167:26981.

23. Hu J, Lin W, Lin B, Wu K, Fan H, Yu Y. Persistent DNA methylation changes in zebrafish following graphene quantum dots exposure in surface chemistry-dependent manner. Ecotoxicol Environ Saf. 2019;169:370-5.

24. Deng S, Jia PP, Zhang JH, Junaid M, Niu A, Ma YB, Fu A, Pei DS. Transcriptomic response and perturbation of toxicity pathways in zebrafish larvae after exposure to graphene quantum dots (GQDs). J Hazard Mater. 2018;357:146-58.

25. Xu L, Zhao J, Wang Z. Genotoxic response and damage recovery of macrophages to graphene quantum dots. Science of The Total Environment. 2019;664:536-45.

26. Wu T, Li Y, Liang X, Liu X, Tang M. Identification of potential circRNA-miRNA-mRNA regulatory networks in response to graphene quantum dots in microglia by microarray analysis. Ecotoxicol Environ Saf. 2021;208:111672.

27. Wang T, Zhu S, Jiang X. Toxicity mechanism of graphene oxide and nitrogen-doped graphene quantum dots in RBCs revealed by surface-enhanced infrared absorption spectroscopy. Toxicology Research. 2015;4:885-94.

28. Deng S, Fu A, Junaid M, Wang Y, Yin Q, Fu C, Liu L, Su DS, Bian WP, Pei DS. Nitrogen-doped graphene quantum dots (N-GQDs) perturb redox-sensitive system via the selective inhibition of antioxidant enzyme activities in zebrafish. Biomaterials. 2019;206:61-72.

29. Wu T, Liang X, Liu X, Li Y, Wang Y, Kong L, Tang M. Induction of ferroptosis in response to graphene quantum dots through mitochondrial oxidative stress in microglia. Part Fibre Toxicol. 2020;17:30. 
30. Xu L, Dai Y, Wang Z, Zhao J, Li F, White JC, Xing B. Graphene quantum dots in alveolar macrophage: uptake-exocytosis, accumulation in nuclei, nuclear responses and DNA cleavage. Part Fibre Toxicol. 2018;15:45.

31. Chong Y, Ma Y, Shen H, Tu X, Zhou X, Xu J, Dai J, Fan S, Zhang Z. The in vitro and in vivo toxicity of graphene quantum dots. Biomaterials. 2014;35:5041-8.

32. Zhang D, Zhang Z, Wu Y, Fu K, Chen Y, Li W, Chu M. Systematic evaluation of graphene quantum dot toxicity to male mouse sexual behaviors, reproductive and offspring health. Biomaterials. 2019;194:215-32.

33. Nurunnabi M, Khatun Z, Huh KM, Park SY, Lee DY, Cho KJ, Lee YK. In vivo biodistribution and toxicology of carboxylated graphene quantum dots. ACS Nano. 2013;7:6858-67.

34. Chakraborty C, Sharma AR, Sharma G, Lee SS. Zebrafish: A complete animal model to enumerate the nanoparticle toxicity. J Nanobiotechnology. 2016;14:65.

35. Wang Y, Hamid N, Jia P-P, Pei D-S: A comprehensive review on genetically modified fish: key techniques, applications and future prospects. Reviews in Aquaculture 2021, n/a.

36. Chakraborty $\mathrm{C}$, Hsu CH, Wen ZH, Lin CS, Agoramoorthy G. Zebrafish: a complete animal model for in vivo drug discovery and development. Curr Drug Metab. 2009;10:116-24.

37. Cheng J, Flahaut E, Cheng SH. Effect of carbon nanotubes on developing zebrafish (Danio rerio) embryos. Environ Toxicol Chem. 2007;26:708-16.

38. Usenko CY, Harper SL, Tanguay RL. In vivo evaluation of carbon fullerene toxicity using embryonic zebrafish. Carbon N Y. 2007;45:1891-8.

39. Manjunatha B, Park SH, Kim K, Kundapur RR, Lee SJ. In vivo toxicity evaluation of pristine graphene in developing zebrafish (Danio rerio) embryos. Environ Sci Pollut Res Int. 2018;25:12821-9.

40. Chen Y, Hu X, Sun J, Zhou Q. Specific nanotoxicity of graphene oxide during zebrafish embryogenesis. Nanotoxicology. 2016;10:42-52.

41. Chousidis I, Stalikas CD, Leonardos ID. Induced toxicity in early-life stage zebrafish (Danio rerio) and its behavioral analysis after exposure to non-doped, nitrogen-doped and nitrogen, sulfur-co doped carbon quantum dots. Environ Toxicol Pharmacol. 2020;79:103426.

42. Zhang JH, Sun T, Niu A, Tang YM, Deng S, Luo W, Xu Q, Wei D, Pei DS. Perturbation effect of reduced graphene oxide quantum dots (rGOQDs) on aryl hydrocarbon receptor (AhR) pathway in zebrafish. Biomaterials. 2017;133:49-59.

43. Detrich HW, Detrich HW III, Westerfield M, Zon LI. The zebrafish: genetics, genomics and informatics. Academic Press; 2011.

44. Zhao YY, Qu SN, Feng XY, Xu JC, Yang Y, Su SC, Wang SP, Ng KW. Tailoring the Photoluminescence Excitation Dependence of the Carbon Dots via an Alkali Treatment. J Phys Chem Lett. 2019;10:4596-602.

45. Liu Y, Zhu C, Gao Y, Yang L, Xu J, Zhang X, Lu C, Wang Y, Zhu Y. Biomass-derived nitrogen self-doped carbon dots via a simple one-pot method: Physicochemical, structural, and luminescence properties. 
Appl Surf Sci. 2020;510:145437.

46. Wang Y, Li Y, Xu Y. Synthesis of mechanical responsive carbon dots with fluorescence enhancement. J Colloid Interface Sci. 2020;560:85-90.

47. Sottile ML, Nadin SB. Heat shock proteins and DNA repair mechanisms: an updated overview. Cell Stress Chaperones. 2018;23:303-15.

48. Wang D, Zhu L, Chen JF, Dai L. Can graphene quantum dots cause DNA damage in cells? Nanoscale 2015, 7:9894-9901.

49. Derr JB, Tamayo J, Clark JA, Morales M, Mayther MF, Espinoza EM, Rybicka-Jasinska K, Vullev VI. Multifaceted aspects of charge transfer. Phys Chem Chem Phys. 2020;22:21583-629.

50. Oschman JL. Charge transfer in the living matrix. J Bodyw Mov Ther. 2009;13:215-28.

51. Park EJ, Lee SJ, Lee K, Choi YC, Lee BS, Lee GH, Kim DW. Pulmonary persistence of graphene nanoplatelets may disturb physiological and immunological homeostasis. J Appl Toxicol. 2017;37:296-309.

52. Bramini M, Sacchetti S, Armirotti A, Rocchi A, Vazquez E, Leon Castellanos V, Bandiera T, Cesca F, Benfenati F. Graphene Oxide Nanosheets Disrupt Lipid Composition, $\mathrm{Ca}(2+)$ Homeostasis, and Synaptic Transmission in Primary Cortical Neurons. ACS Nano. 2016;10:7154-71.

53. Rudy Y. Molecular basis of cardiac action potential repolarization. Ann N Y Acad Sci. 2008;1123:113-8.

54. Kitazono T, Faraci FM, Taguchi H, Heistad DD. Role of potassium channels in cerebral blood vessels. Stroke. 1995;26:1713-23.

55. Delisle BP, Anson BD, Rajamani S, January CT. Biology of cardiac arrhythmias: ion channel protein trafficking. Circ Res. 2004;94:1418-28.

56. Nessa A, Rahman SA, Hussain K. Hyperinsulinemic Hypoglycemia - The Molecular Mechanisms. Frontiers in Endocrinology. 2016;7:29.

57. Nieves-Cintron M, Flores-Tamez VA, Le T, Baudel MM, Navedo MF. Cellular and molecular effects of hyperglycemia on ion channels in vascular smooth muscle. Cell Mol Life Sci. 2021;78:31-61.

58. Zou W, Zhou Q, Zhang X, Mu L, Hu X. Characterization of the effects of trace concentrations of graphene oxide on zebrafish larvae through proteomic and standard methods. Ecotoxicol Environ Saf. 2018;159:221-31.

59. Liao Y, Wang W, Li Z, Wang Y, Zhang L, Huang X, Cai P: Comparative proteomic analysis reveals cytotoxicity induced by graphene oxide exposure in A549 cells. $J$ App/ Toxico/2020, n/a.

60. Eymin B. Targeting the spliceosome machinery: A new therapeutic axis in cancer? Biochem Pharmacol 2020:114039.

61. Wu Q, Zhao Y, Zhao G, Wang D. microRNAs control of in vivo toxicity from graphene oxide in Caenorhabditis elegans. Nanomedicine. 2014;10:1401-10.

62. Zhao Y, Wu Q, Wang D. A microRNAs-mRNAs network involved in the control of graphene oxide toxicity in Caenorhabditis elegans. RSC Advances. 2015;5:92394-405. 
63. Nabeshima Y, Hanaoka K, Hayasaka M, Esumi E, Li S, Nonaka I, Nabeshima Y. Myogenin gene disruption results in perinatal lethality because of severe muscle defect. Nature. 1993;364:532-5.

\section{Figures}

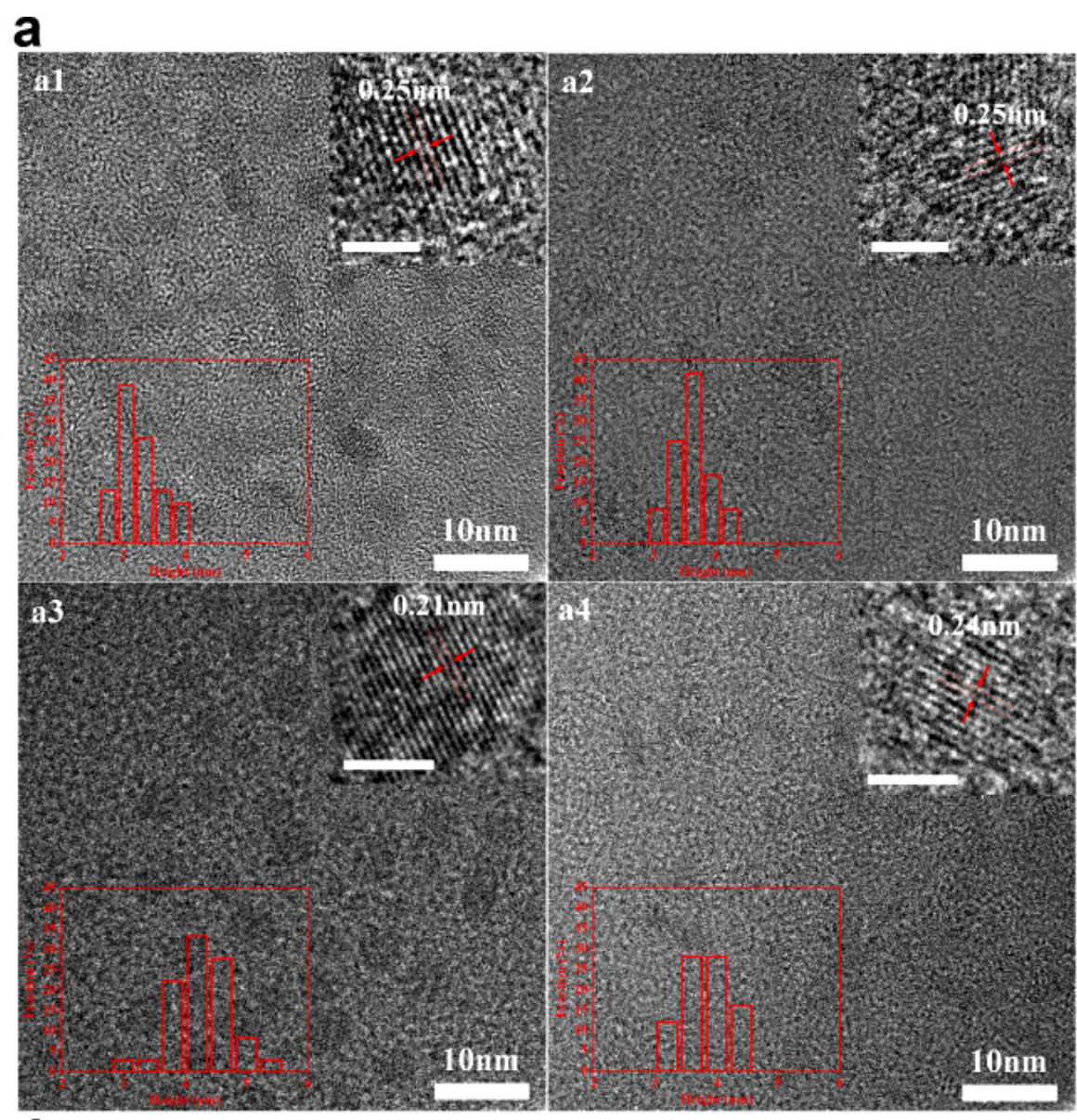

b

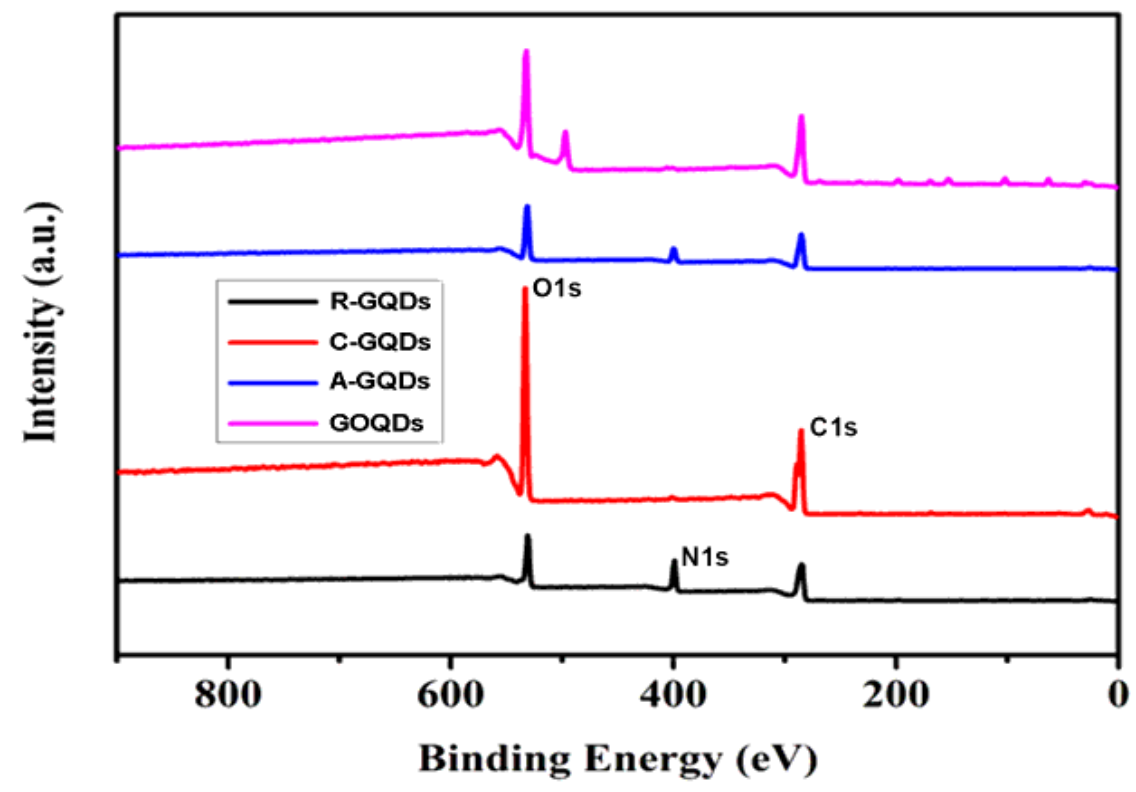

Figure 1 
Surface Characterization of GQDs. (a) TEM image of R-GQDs (a1), GOQDs (a2), C-GQDs (a3), and AGQDs (a4). Insert shows the size distribition and high resolution TEM (HRTEM) of the corresponding GQDs, The scale bar in HRTEM is 2nm. (b) The wide-scan XPS spectra for GQDs and three elemental peaks (N1s, C1s, and 01s) are marked in the spectra profiles.

a

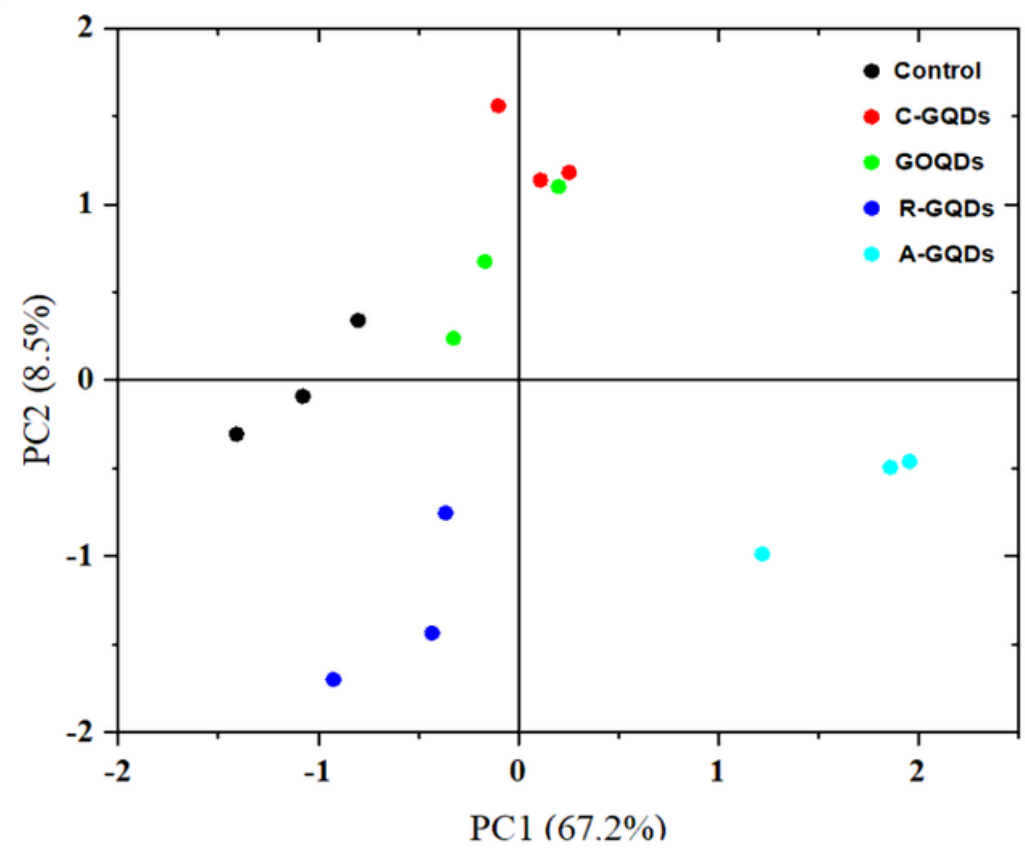

b

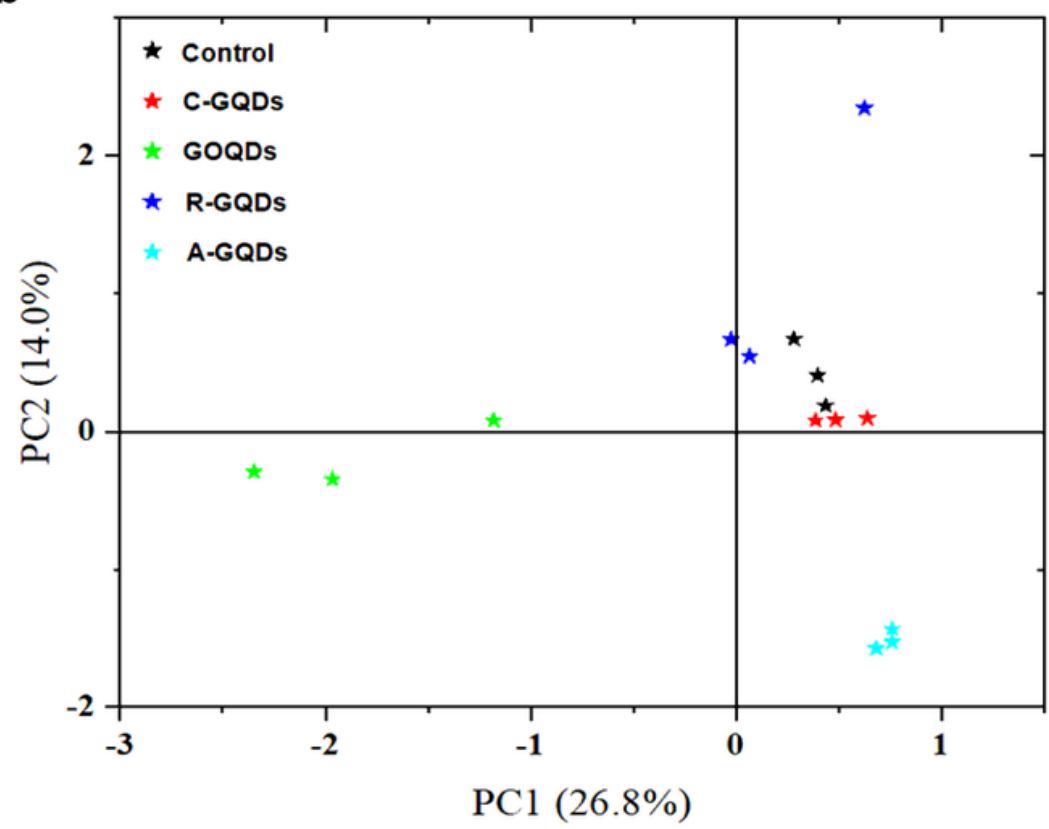

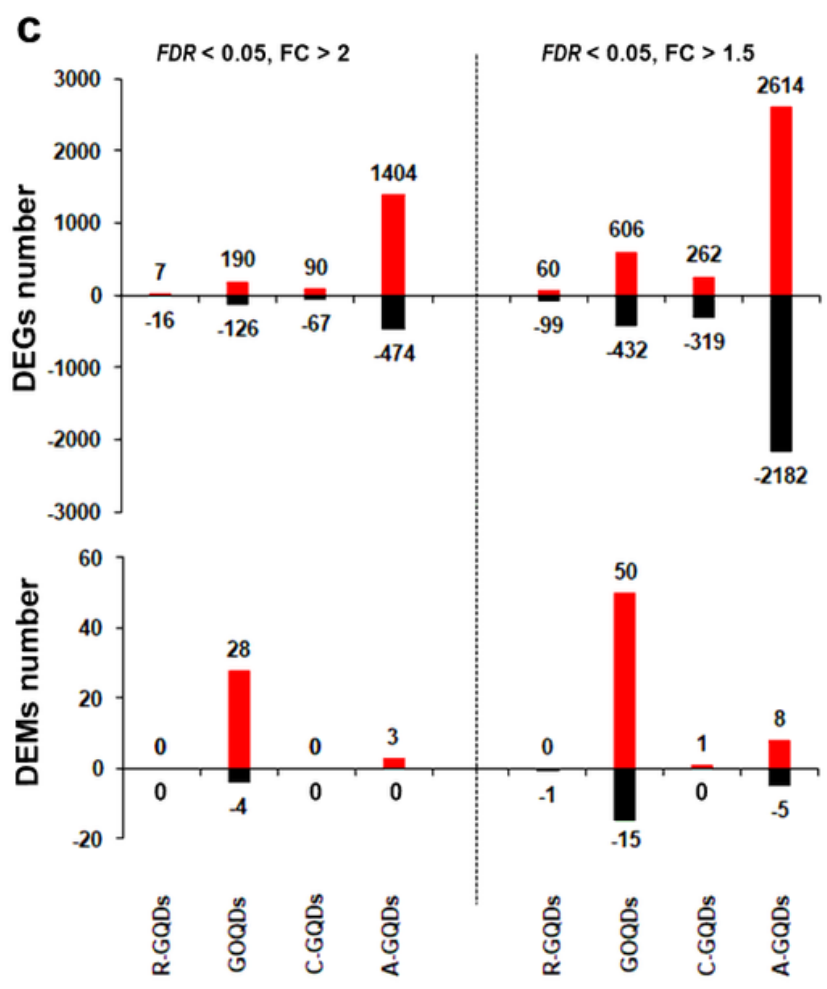

d

CK vs GOQDs $\odot$ CK vs A-GQDs $\odot$ CK vs R-GQDs $\odot$ CK vs C-GQDs

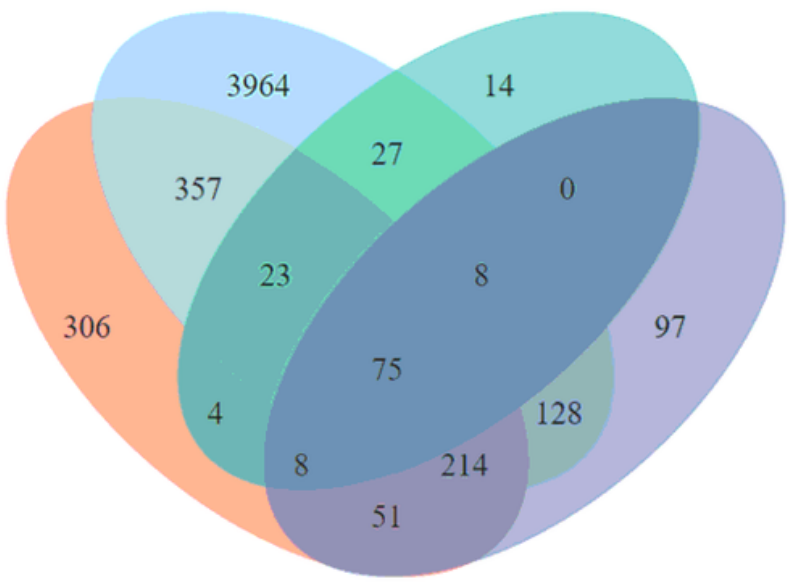

Figure 2

RNA-seq data analysis. PCA analysis of (a) mRNAs and (b) miRNAs expression profiling among the four GQDs treatments, respectively. (c) Statistical results of differentially expressed mRNA (DEGs) and miRNAs (DEMs) between control and different GQDs treatment, red and black column represents the upand down-regulated mRNAs or miRNAs. (d) Venn analysis of the DEGs from various GQDs treatment based on a FDR value of $<0.05$ and a fold change (FC) of $>1.5$. 
a

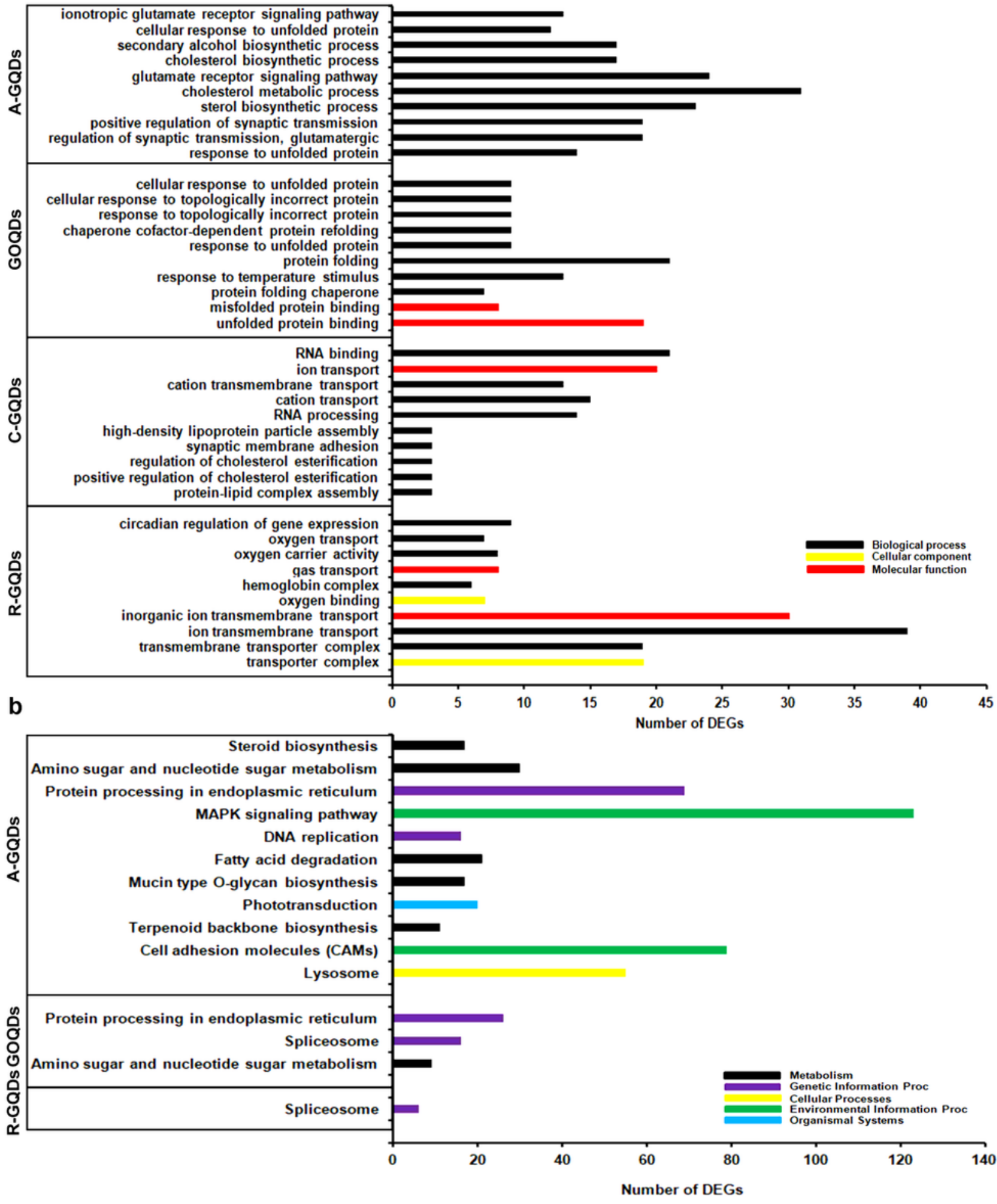

Figure 3

Functional enrichment analysis for DEGs induced by various GQDs treatment. (a) The top ten GO terms and (b) KEGG pathways generalized from each GQDs treatment, both were screened out with a FDR of < 0.05 . 
a
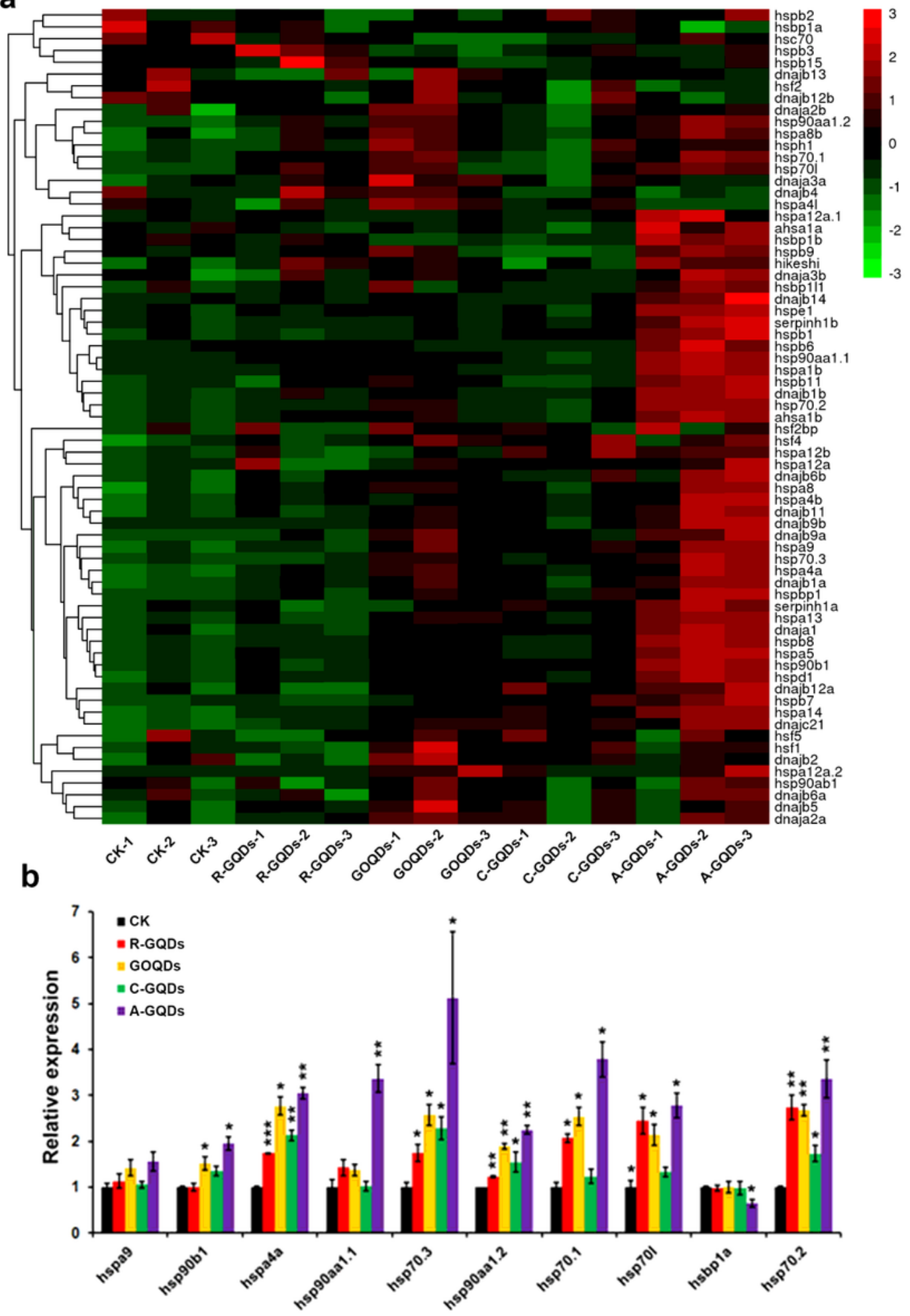

Figure 4

(a) Heatmap exhibition of the expression abundance of HSP family members across all samples among five treatments, the logarithmic of fold change (base 2) is depicted according to the color scale. (b) qPCR verifcation of the expression changes of ten hsp genes in the four GQDs treated groups. Data are represented by Mean \pm SD with four independent biological replicates, a $p$ value of $<0.05$ is considered to be significant. 
a

\section{d}
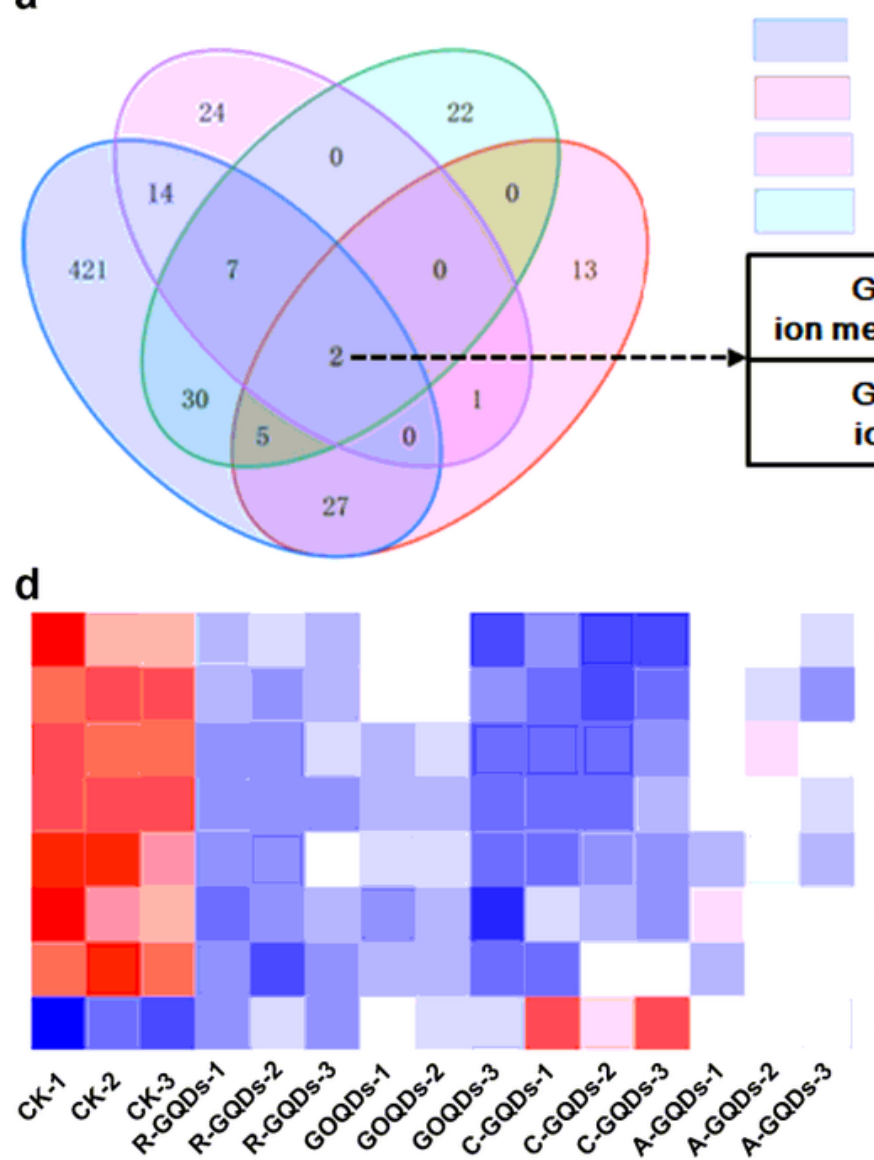

e

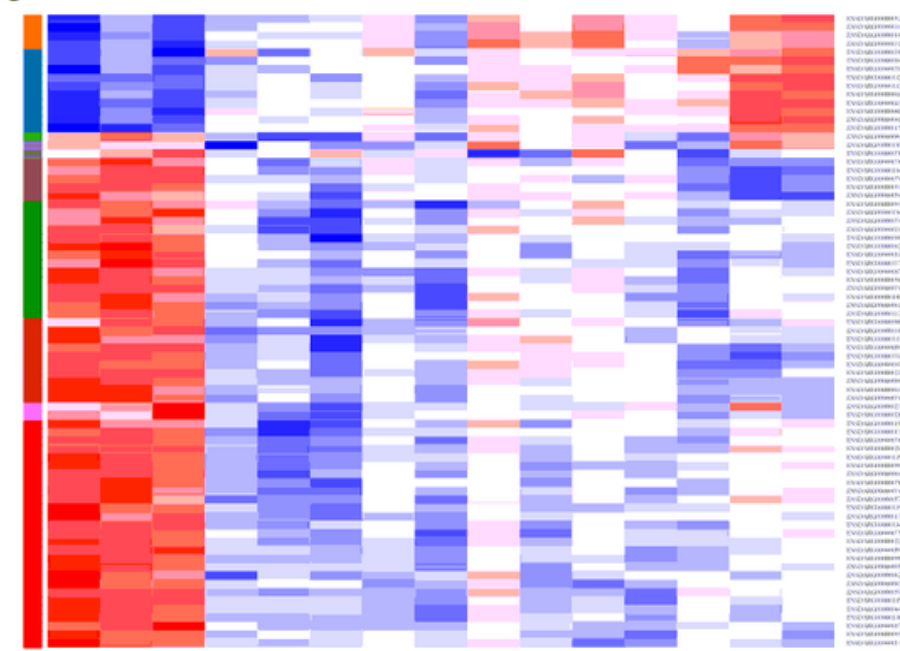

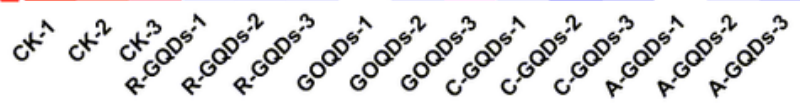

b

CKvs A-GQDs

CKvs GOQDs

CKvs C-GOQDs

CK vs R-GQDs

GO:0034220

GO:0006811

ion transport

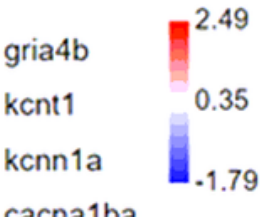

cacna1ba

kcnma1a

cacna1db

abcc5

apoa 4 b. 2

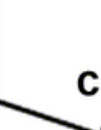

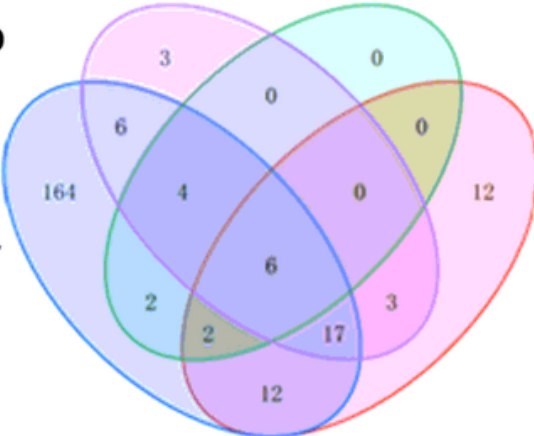

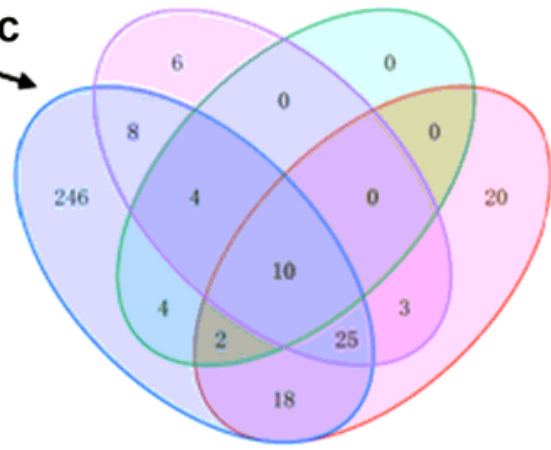

f

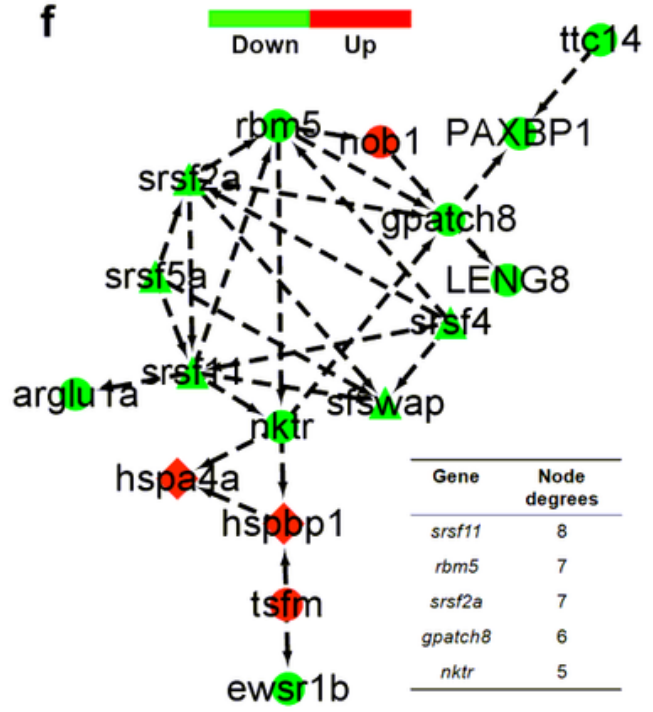

\section{Figure 5}

Analysis of the common gene clusters and associated signaling pathways induced by the four GQDs treatments. (a) Venn analysis based on the significantly enriched GO terms from respetive GQDs treatment. Venn analysis of the number of the overlapping DEGs induced in the GO term- (b) 'ion membrane transport' and (c) 'ion transport', respectively. d) A summary of the overlapping genes screened out from (b) and (c) depicted by heatmap. (e) The common signaling pathways shared by the 
four GQDs treatments, which are extracted from the online String database and drawn from Cytoscape 3.8.0.

\section{RNA-seq $\square$ qPCR}

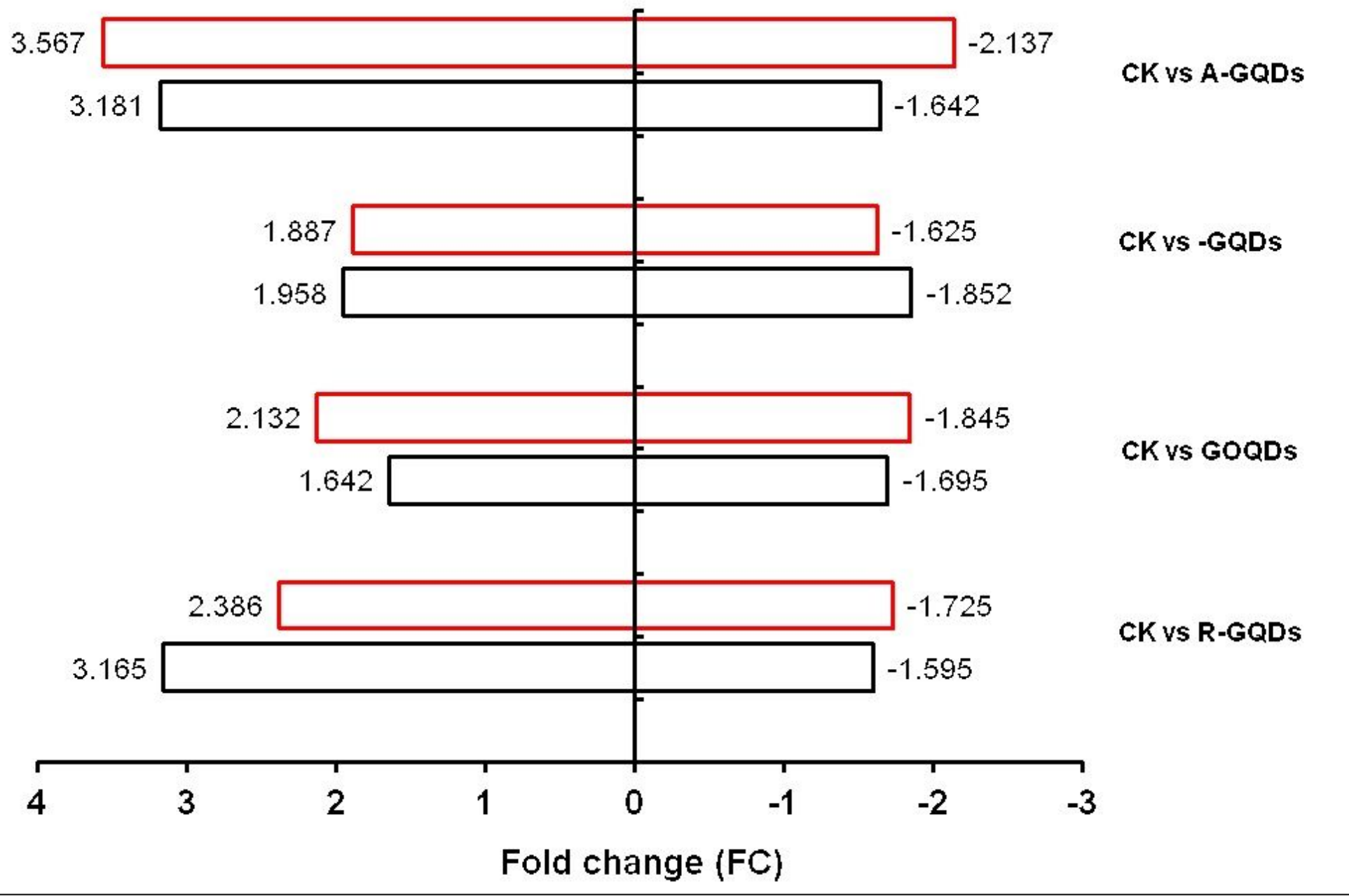

\section{Figure 6}

Comparion beween RNA-seq data and qPCR results for dre-miR-735-5p and its target gene - myog, respectively shown in the left and right columns. QPCR is performed with four independent biological replicates, and only the average values $(p<0.05)$ are shown in the figure.

\section{Supplementary Files}

This is a list of supplementary files associated with this preprint. Click to download.

- OnlineGraphicAbstract.png

- Additionalfiles.rar 